\title{
Virtual Screening for Finding Novel COX-2 Inhibitors as Antitumor Agents
}

\author{
Zohreh S. Badieyan ${ }^{1,4}$, Seyed Adel Moallem*,2,3,4 ${ }^{*}$ Soghra Mehri ${ }^{4}$, Shabnam Shahsavand ${ }^{4}$, and \\ Farzin Hadizadeh* ${ }^{* 1,4}$
}

${ }^{I}$ Biotechnology Research Center, Mashhad University of Medical Sciences, Mashhad, Iran; ${ }^{2}$ Pharmaceutical Sciences Research Center, Mashhad University of Medical Sciences, Mashhad, Iran; ${ }^{3}$ Medical Toxicology Research Center, Mashhad University of Medical Sciences, Mashhad, Iran; ${ }^{4}$ School of Pharmacy, Mashhad University of Medical Sciences, Mashhad, Iran

\begin{abstract}
The cyclooxygenase-2 (COX-2) enzyme binds to arachidonic acid resulting in the release of metabolites that induce pain and inflammatory responses. Recent studies have shown that strong COX-2 expression is highly correlated with increased tumor risk. Therefore, the development of potent COX-2 inhibitors to relieve pain and treat cancers requires further investigation. We used virtual screening to find three COX-2 inhibitors (Phar-95239, T0511-4424 and Zu4280011) from a huge zinc database containing 2000000 compounds. The effects of the compounds on COX-2 were compared to those on COX-1 using a colorimetric COX (ovine) screening assay kit. The selectivity index, the ratio of $\mathrm{IC}_{50}$ for COX-1 inhibition to that of COX-2, calculated were MTT assay was used to evaluate the cytotoxic activity of the compounds using different dilutions. The $\mathrm{IC}_{50}$ values were calculated. Based on the results of the MTT assay, the $\mathrm{IC}_{50}$ values for compounds Phar-95239, T0511-4424 and $\mathrm{Zu}-4280011$ were 178.52, 143 and $97.61 \mu \mathrm{M}$, respectively, and the selectivity indices of the compounds were $11.36,12.20$ and 20.03 , respectively. These results indicated a relationship between the selectivity index and anticancer activity. $\mathrm{Zu}-4280011$ displayed the highest selectivity index and the best results in the MTT assay among selected componds.
\end{abstract}

Keywords: Cyclooxygenase, MTT, Selectivity index, Virtual Screening, Zinc database.

\section{INTRODUCTION}

The use of nonsteroidal anti-inflammatory drugs (NSAIDs) for the treatment of inflammation and pain is often accompanied by adverse gastrointestinal and renal side effects [1]. The anti-inflammatory activities of these drugs are mediated by the inhibition of cyclooxygenases (COXs), which catalyze the bioconversion of arachidonic acid to prostaglandins[2]. However, the inhibition of COXs may lead to undesirable side effects. Currently, it is well established that there are at least two COX isozymes, COX-1 and COX-2 [3]. The constitutively expressed COX-1 isozyme is produced in a variety of tissues and appears to be important for the maintenance of physiological functions such as gastroprotection and vascular homeostasis. On the other hand, the COX-2 isozyme is induced by mitogenic and proinflammatory stimuli, suggesting the involvement of this isozyme in inflammatory processes. Therefore, the selective inhibition of COX-2, but not COX-1 is useful for treating inflammation and inflammation-associated disorders. COX-2

*Address correspondence to these authors at the Biotechnology Research Center, Mashhad University of Medical Sciences, Mashhad, Iran, and School of Pharmacy, Mashhad University of Medical Sciences, Mashhad, Iran; Tel: 0098-511-8823252; Fax: 0098-511-8823251;

E-mails: moallem@mums.ac.ir; hadizadehf@mums.ac.ir inhibitors also have lower gastrointestinal toxicities than other NSAIDs [4]. Recent studies have shown that the progression of Alzheimer's disease is reduced among some users of NSAIDs. Chronic treatment with selective COX-2 inhibitors may slow the progress of Alzheimer's disease without causing gastrointestinal damage $[5,6]$. Therefore, selective COX-2 inhibitors have been developed as a new generation of NSAIDs with diminished GI side effects. However, rofecoxib and valdecoxib, which are highly selective COX-2 inhibitors, have been withdrawn from the market due to an increased risk of cardiovascular complications. COX-2 mediates the biosynthesis of prostacyclin which is a vasodilator and an inhibitor of platelet aggregation. The indirect inhibition of prostacyclin production by selective COX2 inhibitors might account for their adverse cardiovascular effects $[7,8]$.

In addition to the role of COX-2 in rheumatoid arthritis and osteoarthritis, COX-2 expression is triggered by inflammation and carcinogenesis. [4] COX-2 is overexpressed in many solid tumors such as colon [9], breast [10], prostate [11], liver [12] and lung [3] cancers. COX-2 inhibition in vitro using specific COX-2 inhibitors has demonstrated that COX-2 is a potential target for novel cancer therapies [13, 14]. Research attempts to discover selective COX-2 inhibitors have produced many classes of compounds such as 
coxibs that possess the desired selectivity. For this reason, novel scaffolds with high selectivity for COX-2 inhibition must be identified and evaluated for their anticancer effects [15].

Virtual screening (VS) is a computational technique that is used in drug discovery research and involves the rapid in silico assessment of large libraries of chemical structures to identify structures that will most likely bind to a drug target such as a protein receptor or enzyme $[16,17]$.

As part of our program to discover novel types of selective COX-2 inhibitors, we now report a methodology to find a new group of COX-2 inhibitory compounds and evaluate their anti-inflammatory and anticancer activities [18].

\section{MATERIALS AND METHODOLOGY}

\section{In Silico Studies}

Initially, we downloaded approximately 2000000 compounds from the ZINC database (http://zinc.docking.org/). Approximately 12000 compounds with a structure similar to that of the selective COX-2 inhibitor, celecoxib, were selected using ChemOffice (www.cambridgesoft.com). A structure-based virtual screening approach was used to search for new COX-2 inhibitors. The virtual screening was performed using the crystal structure of mouse COX-2 bound to SC-558, which is a COX-2-selective inhibitor (PDB code 6COX) [19]. The ZINC database (using 12000 commercially available compounds) was computationally screened based on binding to the active site of COX-2 using the AutoDock 3.5 program [20]. The calculation by virtual screening took approximately 2.5 days to run on Linux cluster including six Pentium 3-GHz CPUs.

The 40 top-scoring compounds were visually inspected for the plausibility of their predicted binding modes. We selected three of the best compounds with lower docking energies for biological testing.

\section{In Vitro Cyclooxygenase (COX) Inhibition Assay}

The ability of the test compounds to inhibit ovine COX-1 and COX-2 was determined using a colorimetric COX (ovine) inhibitor-screening assay (Cayman Chemical, Item Number 760111) that utilizes the peroxidase component of cyclooxygenase. The peroxidase activity is colorimetrically assayed by monitoring the appearance of oxidized $\mathrm{N}, \mathrm{N}, \mathrm{N}^{\prime}, \mathrm{N}$ '-tetramethyl-p-phenylenediamine (TMPD) at 590 $\mathrm{nm}[1]$.

\section{Cell Line and Culture}

The human breast cancer cell line, MCF-7, was obtained from the Pasteur Institute (Tehran, Iran). Cells were maintained at $37^{\circ} \mathrm{C}$ in a humidified atmosphere $(95 \%)$ containing $5 \% \mathrm{CO}_{2}$. Different cell lines were cultured in Dulbecco's Modified Eagle's Medium (DMEM) supplemented with $10 \%$ $\mathrm{v} / \mathrm{v}$ fetal bovine serum, 100 units/ml of penicillin and 100 $\mu \mathrm{g} / \mathrm{ml}$ of streptomycin.

\section{MTT Assay}

The MTT [3-(4,5-Dimethylthiazol-2-yl)-2,5-diphenyltetrazolium bromide] method was performed to assess the cy- totoxicity of different compounds [21,22]. Briefly, 10000 MCF-7 cells were seeded into 96-well plates and cultured overnight. The cells were treated with different concentrations of the test compounds $(1-200 \mu \mathrm{M})$. After $24 \mathrm{~h}, 20 \mu \mathrm{l}$ of MTT was added to each well to give a final concentration of $0.5 \mathrm{mg} / \mathrm{ml}$, and the cells were incubated for $3 \mathrm{~h}$ at $37^{\circ} \mathrm{C}$. The formazan dye was solubilized with DMSO. The absorbance was measured at $545 \mathrm{~nm}(630 \mathrm{~nm}$ as a reference) in an ELISA reader (Start Fax-2100, UK).

\section{RESULTS}

\section{In Silico Studies}

We used virtual screening to select three compounds (Phar-095239, Zu-4280011, T0511-4424) with lower docking energies. Celecoxib and SC-558 were used as positive controls (Fig. 1).

Docking view of compounds Phar-095239 (a), T05114424 (b), $\mathrm{Zu}-4280011$ (c), and celecoxib (d) at the COX-2 active site has been shown in Fig. (2). The docking energy of the compounds (approximately $-14 \mathrm{kcal} / \mathrm{mol}$ ) was higher than that of the positive controls: SC-558 $(-10.38 \mathrm{kcal} / \mathrm{mol})$ and celecoxib $(-8.02 \mathrm{kcal} / \mathrm{mol})$.

\section{Cyclooxygenase Inhibition Studies}

The test compounds (Phar-95239, T0511-4424, Zu4280011) were subjected to in vitro cyclooxygenase (COX) inhibition assays (Table 1). Concentration-COX-2 activity inhibition curve of three test compounds and celecoxib are shown in Fig. (3). The $\mathrm{IC}_{50}$, the concentration of compounds that provided $50 \%$ inhibition of COX-2 and COX-1, was calculated. $\mathrm{IC}_{50} \mathrm{~S}$ of the test compounds (Phar-95239, T05114424, Zu-4280011) and celecoxib for inhibition of COX-1 were $9.32,8.42,15.23$ and $13.02 \mu \mathrm{M}$, respectively. $\mathrm{IC}_{50}$ values for COX-2 inhibition were found to be $0.82,0.69,0.76$ and $0.49 \mu \mathrm{M}$, respectively. Selectivity indices or the ratio of $\mathrm{IC}_{50}$ for COX-1 inhibition to that of COX-2 of the test compounds and celecoxib were 11.36, $12.20,20.03$ and 26.57, respectively. The results are shown in Table 1.

The compound $\mathrm{Zu}-4280011$ displayed a high selectivity index of 20.03 near that of celecoxib (26.57) and inhibited COX-2 activity to an extent similar to that of celecoxib.

\section{Effects of the Test Compounds on Cell Viability}

Different concentrations of the test compounds (1-200 $\mu \mathrm{M})$ were used in the MTT assay on MCF-7 cells. The compounds decreased cell viability in a concentration dependent manner (Fig. 4). $\mathrm{IC}_{50}$ for MCF-7 growth inhibition of celecoxib and test compounds (Phar-95239, T0511-4424, Zu4280011) were 87.69, 178.52, 143 and 97.61, respectively (Table 1). Zu-4280011 was the most potent compound second to celecoxib.

\section{DISCUSSION AND CONCLUSION}

We used virtual screening to find three COX-2 inhibitors from a huge zinc database (2000000 compounds). According to docking studies, we predicted that $\mathrm{IC}_{50}$ values of these compounds for inhibition of $\mathrm{COX}-2$ were less than celecoxib. Although the calculated $\mathrm{IC}_{50}$ values were not exactly what we expected, finding three potent $\mathrm{COX}-2$ inhibitors 

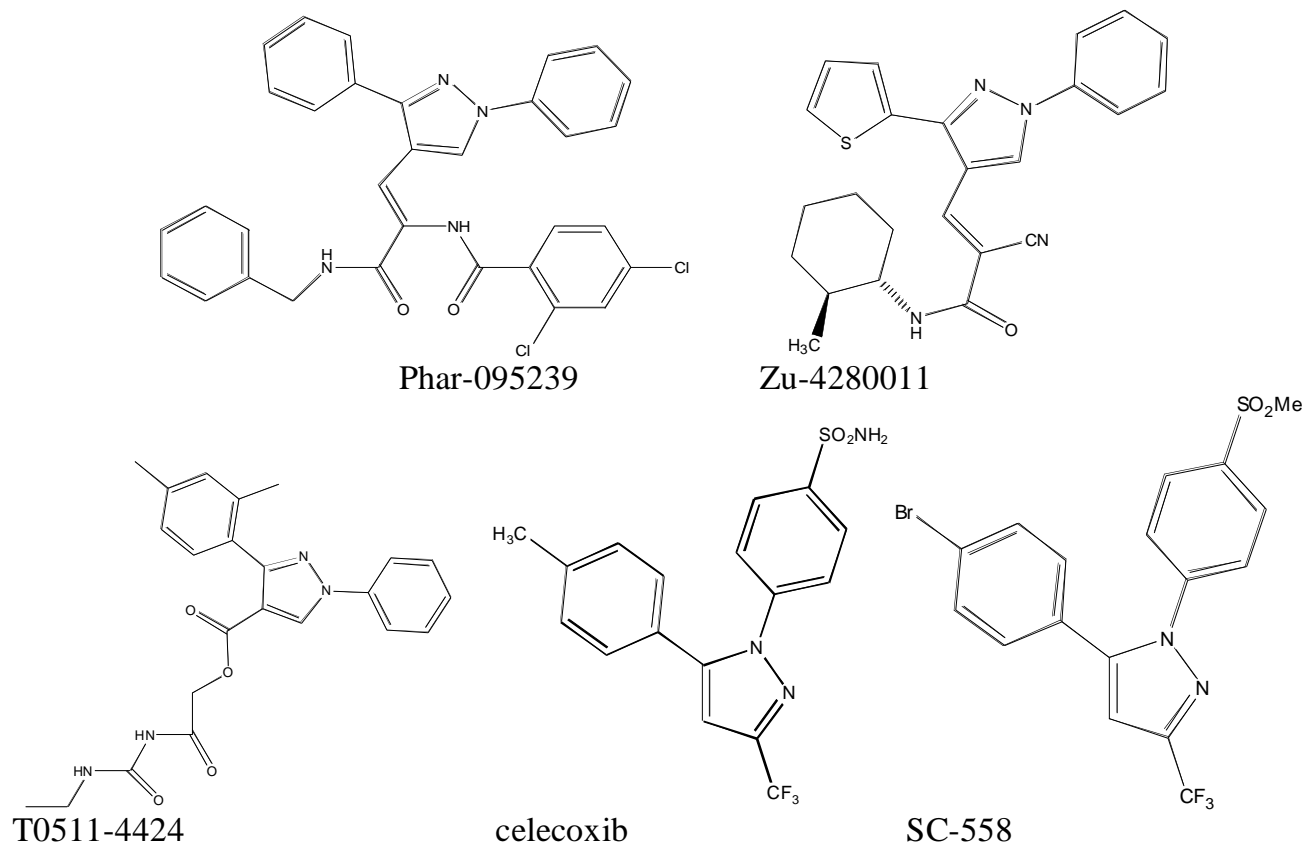

SC-558

Fig. (1). Chemical structure of the test compounds (Phar-95239, T0511-4424, Zu-4280011) and positive controls (celecoxib and SC-558).

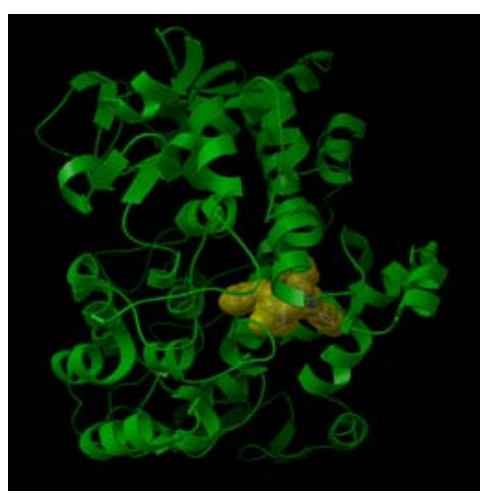

(a)

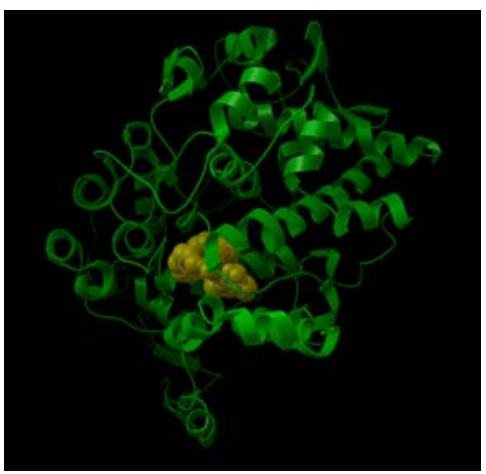

(c)

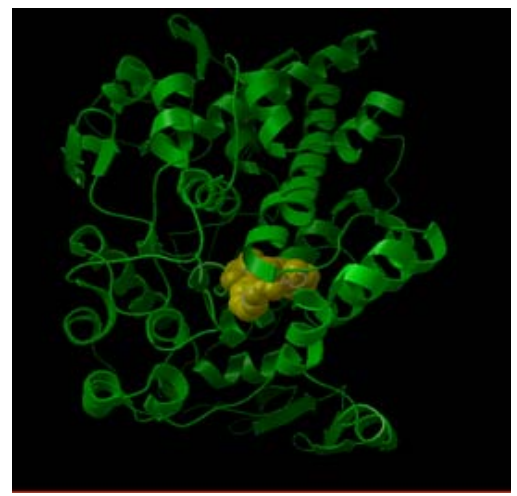

(b)

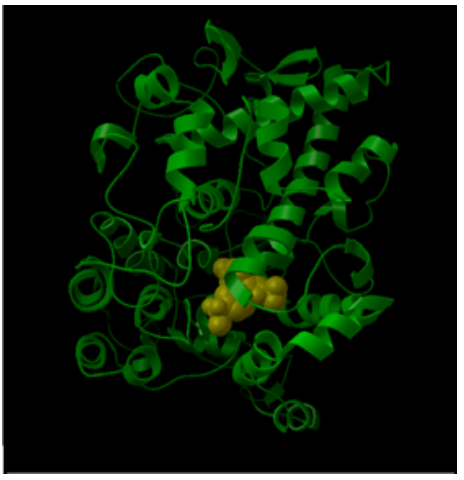

(d)

Fig. (2). Docking view of compounds Phar-095239 (a), T0511-4424 (b), Zu-4280011 (c), and celecoxib (d) at COX-2 active site.

among a large database was a remarkable achievement, thanks to virtual screening.
We compared the COX-2 $\mathrm{IC}_{50}$ values with MTT assay results (Table 1). We concluded that the order of the inhibitory effects of compounds on MCF-7 cell line proliferation 


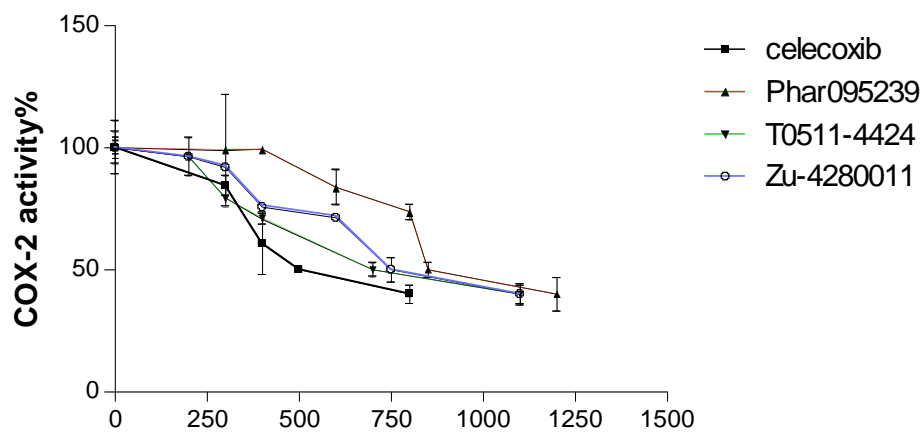

Fig. (3). Concentration-COX-2 activity inhibition curve of three test compounds and celecoxib.

Table 1. Inhibitory Effects of the Test Compounds on COX Enzymes and MCF-7 Cells Proliferation

\begin{tabular}{|c|c|c|c|c|c|}
\hline \multirow{2}{*}{ MTT assay } & \multirow{2}{*}{$\mathbf{I C}_{\mathbf{5 0}}(\boldsymbol{\mu M})$} & $\mathbf{C O X}-\mathbf{2}$ & $\mathbf{C O X}-\mathbf{1}$ & \multirow{2}{*}{ ZINC code* } & \multirow{2}{*}{ Compounds name } \\
\hline \hline 87.69 & 26.57 & $\mathbf{I C}_{\mathbf{5 0}}(\boldsymbol{\mu M})$ & $\mathbf{I C}_{\mathbf{5 0}}(\boldsymbol{\mu M})$ & - & Celecoxib \\
\cline { 3 - 5 } & 11.36 & 0.49 & 13.02 & 01793979 & $\mathrm{Phar}-095239$ \\
\hline 178.52 & 12.20 & 0.82 & 9.32 & 05446539 & $\mathrm{~T} 0511-4424$ \\
\hline 143 & 20.03 & 0.69 & 8.42 & 05036105 & $\mathrm{Zu}-4280011$ \\
\hline
\end{tabular}

*This code is used to access compounds in zinc database.

$* *$ Selectivity index $=\frac{\text { IC50 for inhibition of } \operatorname{COX}-1}{\text { IC50 for inhibition of } \operatorname{COX}-2}$

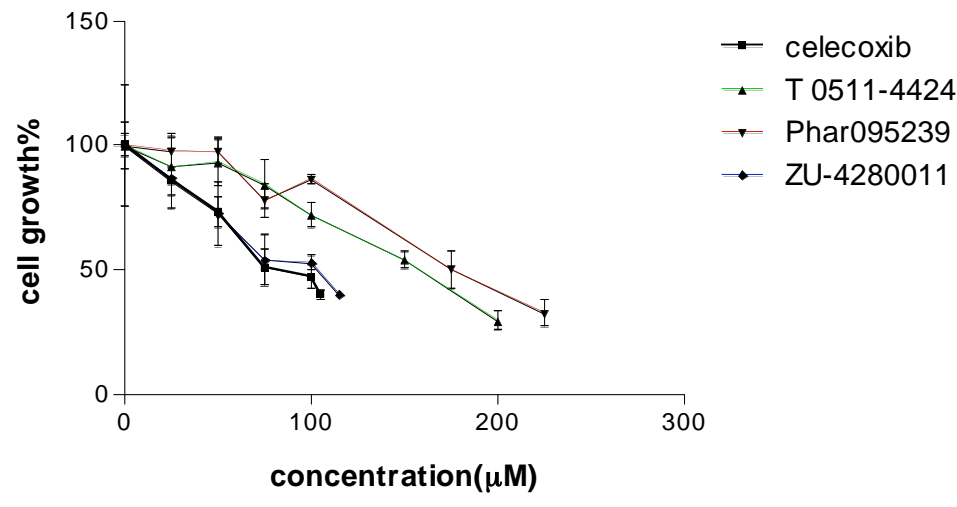

Fig. (4). Concentration-cell growth inhibition curve of three test compounds and celecoxib. Cell growth inhibition was measured by the MTT assay.

did not correlate with their inhibitory effect on COX-2 enzyme. The order of $\mathrm{IC}_{50}$ values for $\mathrm{COX}-2$ inhibition was: celecoxib < T0511-4424 < Zu-4280011 < Phar-095239 and the $\mathrm{IC}_{50}$ values for MTT assay was: celecoxib $<\mathrm{Zu}-4280011$ $<$ T0511-4424 < Phar-095239.

Our results demonstrated a relationship between the order of selectivity index and MTT assay results. As the selectivity index values increased, the $\mathrm{IC}_{50}$ values in the MTT assay decreased. In other words, more selective compounds had more growth inhibitory effects. The order of selectivity index was celecoxib >Zu-4280011 > T05114424 > Phar-
095239 and that of $\mathrm{IC}_{50}$ values for MTT assay was Phar$095239>$ T0511-4424 >Zu-4280011> celecoxib. Among these compounds, $\mathrm{Zu}-4280011$ displayed the highest selectivity index (20.03) and the best results in the MTT assay $(97.61 \mu \mathrm{M})$. It can be a candidate for further studies as one potential effective anticancer agent.

We concluded that the compounds' abilities to inhibit proliferation in cancer cells did not significantly correlate with their inhibitory effects on the COX-2 enzyme. The results of the MTT assay demonstrated a relationship between the selectivity index and anticancer activity. As the selectiv- 
ity index increased, the growth inhibitory effects of the tested compounds decreased.

\section{CONFLICT OF INTEREST}

The author(s) confirm that this article content has no conflicts of interest.

\section{ACKNOWLEDGEMENT}

The authors gratefully acknowledge the funding provided by the Vice Chancellor of Research, Mashhad University of Medical Sciences for this project The results described in this paper are part of a PharmD thesis.

\section{REFERENCES}

[1] Avouac, B.; Combe, B.; Darne, B. Prescription of NSAIDs in patients treatment with platelet inhibitors or anticoagulants. Presse. Med., 2003, 32, 38-43.

[2] Surh, Y. J.; Chun, K.S.; Cha, H.H.; Han, S.S.; Keum,Y.S.; Park, K.K.; Lee, S.S. Molecular mechanisms underlying chemopreventive activities of anti-inflammatory phytochemicals: Downregulation of COX-2 and iNOS through suppression of NF-kappa B activation. Mutat. Res., 2001, 480-481, 243-268.

[3] Ermert, L.; Dierkes, C.; Ermert, M. Immunohistochemical expression of cyclooxygenase isoenzymes and downstream enzymes in human lung tumors. Clin. Cancer. Res., 2003, 9, 1604-1610.

[4] Saberi, M.R.; Hadizadeh, F.; Imenshahidi, M.; Shakeri, H.; Ziaee, T.; Ghafuri, M.A.R.; Sakhtianchi. R.; Badieyan, Z.; Hajian, S. Synthesis and Effects of 4,5-Diaryl-2-(2-alkylthio-5-imidazolyl) Imidazoles as Selective Cyclooxygenase Inhibitors. Iran. J. Basic. Med. Sci., 2010, 13, 225-231.

[5] Etminan, M.; Gill, S.; Samii, A. Effect of non-steroidal antiinflammatory drugs on risk of Alzheimer's disease: Systematic review and meta-analysis of observational studies. BMJ, 2003, 327, 128-131.

[6] Weggen, S.; Rogers, M.; Eriksen, J. NSAIDs: small molecules for prevention of Alzheimer's disease or precursors for future drug development? Trends. Pharmacol. Sci., 2007, 28, 536-543.

[7] Bing, R.J.; Lomnicka, M. Why do cyclo-oxygenase-2 inhibitors cause cardiovascular events? J. Am. Coll. Cardiol., 2002, 39, 521522.

[8] Chen, C.S.; Tan, C.M.; Huang, C.H.; Chang, L.C.; Wang, J.P.; Cheng, F.C.; Chern, J.W. Discovery of 3-(4-bromophenyl)-6nitrobenzo[1.3.2]dithiazolium ylide 1,1-dioxide as a novel dual cy- clooxygenase/5-lipoxygenase inhibitor that also inhibits tumor necrosis factor-[alpha] production. Bioorg. Med. Chem., 2010, 18, 597-604.

[9] Marnett, L.J.; DuBois, R.N. COX-2: A target for colon cancer prevention. Annu. Rev. Pharmacol. Toxicol., 2002, 42, 55-80.

[10] Arun, B.; Goss, P. The role of COX-2 inhibition in breast cancer treatment and prevention. Semin. Oncol., 2004, 31, 22-29.

[11] Edwards,J.; Mukherjee, R.; Munro, A.F.; Wells, A.C.; Almushatat A; Bartlett, J.M.S. HER2 and COX2 expression in human prostate cancer. Eur. J. Cancer, 2004, 40, 50-55.

[12] Berasain, C.; Castillo, J.; Perugorria, M.J.; Latasa, M.U.; Prieto, J.; Avila, M.A. Inflammation and liver cancer: new molecular links. Ann. N. Y. Acad. Sci., 2009, 1155, 206-221.

[13] Amir, M.; Agarwal, H.K. Role of COX-2 selective inhibitors for prevention and treatment of cancer. Pharmazie, 2005, 60, 563-570.

[14] Hull, M.A. Cyclooxygenase-2: how good is it as a target for cancer chemoprevention? Eur. J. Cancer, 2005, 41, 1854-1863.

[15] Dubé, D.; Brideau, C.; Deschênes, D.; Fortin, R.; Friesen, W.; Gordon, R.; Girard, Y.; Riendeau, D.; Savoie, C.; Chan,C.C. 2Heterosubstituted-3-(4-methylsulfonyl)phenyl-5-trifluoromethyl pyridines as selective and orally active cyclooxygenase- 2 inhibitors. Bioorg. Med. Chem. Lett., 1999, 9, 1715-1720.

[16] Rester, U. From virtuality to reality - Virtual screening in lead discovery and lead optimization: a medicinal chemistry perspective. Curr. Opin. Drug. Discov., 2008, 11, 559-568.

[17] Rollinger, J.M.; Stuppner, H.; Langer, T. Virtual screening for the discovery of bioactive natural products. Prog. Drug. Res., 2008, 65 , 213-249.

[18] Shoichet, B.K. Virtual screening of chemical libraries. Nature, 2004, 432, 862-865.

[19] Kurumbail, R.G.; Stevens, A.M.; Gierse, J.K.; McDonald, J.J.; Stegeman, R.A.; Pak, J.Y.; Gildehaus, D.; Miyashiro, J.M.; Penning, T.D.; Seibert, K.; Isakson, P.C.; Stallings, W.C. Structural basis for selective inhibition of cyclooxygenase-2 by antiinflammatory agents. Nature, 1996, 384, 644-648.

[20] Morris, G.M.; Goodsell, D.S.; Halliday, R.S.; Huey, R.; Hart, W.E.; Belew, R.K.; Olson, A.J. Automated docking using a Lamarckian genetic algorithm and an empirical binding free energy function. J. Comput. Chem., 1998, 19, 1639-1662.

[21] Mousavi, SH.; Tavakkol-Afshari, J.; Brook, A.; Jafari-Anarkooli, I. Role of caspases and Bax protein in saffron-induced apoptosis in MCF-7 cells. Food. Chem. Toxicol., 2009, 47, 1909-1913.

[22] Tavakkol-Afshari, J.; Brook, A.; Mousavi, S.H. Study of cytotoxic and apoptogenic properties of saffron extract in human cancer cell lines. Food. Chem., 2008, 46, 3443-3447. 